\title{
An improved infrared image processing method based on adaptive threshold denoising
}

\author{
Yu Binbin ${ }^{1,2}$
}

\begin{abstract}
This paper combines the image adaptive threshold denoising algorithm and performs double threshold mapping processing to the infrared image, which effectively reduces the influence of these phenomena to the infrared image and improves the quality of the image. In this paper, the infrared image denoising technology is studied, and an infrared image denoising method based on the wavelet coefficient threshold processing is proposed. This method is based on the noise distribution characteristics of infrared images, the multiplicative noise in the infrared image is transformed into an additive noise, and the wavelet transform coefficient of the transformed infrared image is processed to denoise the image. On this basis, the advantages and disadvantages of the soft and hard threshold functions are deeply analyzed, and an adaptive threshold function with adjustable parameter is constructed. At the same time, in order to suppress the Gibbs visual distortion caused by the absence of translation invariance of the orthogonal wavelet transform, the two-input wavelet transform with translation invariance is introduced, and a double threshold mapping infrared image processing method based on the adaptive threshold denoising algorithm based on the two-input wavelet transform is formed. Simulation results show that the method proposed in this paper has a better suppression of noise, maintains the integrity of image details, and improves the image quality to a certain extent.
\end{abstract}

Keywords: Infrared image, Binary wavelet transform, Image denoising, Threshold function, Double threshold mapping

\section{Introduction}

The infrared image characterizes the thermal properties (radiance) of the point in the scene with the brightness (grayscale) of each pixel. The wavelength of the infrared band is in the range of 0.75 to $1000 \mu \mathrm{m}$, and its shortwavelength boundary is connected to visible light [1]. In theory, the infrared radiation of an object depends on the surface temperature, apparent area, and emissivity $\varepsilon$ of the object, but in fact, the infrared radiation of the surrounding environment (the sky, the ocean, other objects) must be measured, and the environmental factors have complex modulation effects on the infrared radiation of the target. The infrared radiation from the target will be selectively absorbed by some gases in the atmosphere before reaching the infrared sensor, and the

Correspondence: yubb11@mails.ju.edu.cn

${ }^{1}$ College of Computer Science and Technology, Jilin University, Changchun, China

${ }^{2}$ Computer Science and Technology College, Beihua University, Jilin, China airborne particles will also scatter the infrared rays, thereby causing the radiation flux to decay. When the infrared radiation from the target passes through the temperature change region, it will slightly deviate from the original direction. Since this area of heated air is unstable, the radiation deviation is irregular. This effect, called atmospheric flicker, causes the apparent direction and radiant flux of distant targets to change simultaneously.

For complex targets, their surface temperature or radiation flux will be inhomogeneous because of their strange shape and structure and many dispersed heat sources. The target operating speed also has a great influence on its convective cooling rate, which results in the change of target surface temperature. Target surface coating and thermal insulation measures, to a certain extent, can make the target infrared image blurred. Random noise generated by 
sensor elements is also an important obstacle to the transmission of infrared information.

To sum up, the infrared radiation of the target is very complex, which is because the factors affecting the infrared radiation of the target are multifaceted, including the radiation of the target surface, the absorption and reflectivity of the target, the climate conditions, the selective absorption and scattering of the atmosphere, the atmospheric scintillation, the velocity of the target, the surface coating and heat insulation, the noise of the circuit, and so on.

The diversity and complexity of the infrared target image information model determine the infinite dimension characteristics of the infrared target recognition observation sample space, especially the increasing infrared interference and the further development of infrared suppression technology, which makes great difficulties for the recognition of infrared targets. The effectiveness of the target feature strongly depends on the quality of the image preprocessing, which is equivalent to the ability to detect and segment the target effectively in the preprocessing phase of the primary vision processing image (including edge detection, noise filtering [2], image enhancement) under complex background conditions (low signal-to-noise ratio, low contrast, scene change, and so on) [3]. At this time, the processing effect of human vision of the traditional processing method is difficult to achieve, and the processing algorithm with human visual characteristics has a relative advantage. Wavelet transform is an ideal mathematical model for human primary visual information processing [4]. The decomposition of the image signal is not only in the spatial and frequency domains but also in the direction of the information expression local characteristics, which conforms to the basic characteristics of the human vision for the processing of image information. From the spatial domain, the size and direction of some image targets and other details are different, so the image needs to be processed at different resolution and direction. Here, wavelet transform can give full play to its advantages and can be analyzed from the frequency domain. Wavelet transform can decompose all kinds of mixed signals with different frequencies into block signals with different frequencies. Wavelet denoising has the advantages of multiresolution and diversity of wavelet basis selection, which makes it very suitable for image and other signal denoising [5]. Therefore, wavelet transform is a powerful mathematical tool for primary vision processing.

Strictly speaking, speckle noise of infrared image includes both multiplicative noise and additive noise. Aiming at this noise, this paper firstly derives the logarithms of the infrared image and transforms the multiplicative noise into the additive noise. At the same time, an adaptive threshold function with adjustable parameters is constructed. Then, the binary wavelet transform with translation invariance is used for denoising. The specific contributions of this paper can be expressed as follows:

1. To solve the problem of infrared image denoising, an infrared image denoising method based on wavelet coefficient threshold processing is proposed. In this method, the speckle noise of the infrared image is transformed into additive noise by logarithmic transformation, and the threshold of the wavelet coefficients of the transformed infrared image is obtained to realize image denoising.

2. On the basis of infrared image denoising method based on wavelet coefficient threshold processing, the advantages and disadvantages of soft and hard threshold functions are deeply analyzed, and an adaptive threshold function with adjustable parameters is constructed.

3. In order to suppress the Gibbs visual distortion caused by the lack of translation invariance in the orthogonal wavelet transform, the binary wavelet transform with translation invariance is introduced.

4. In the process of infrared image denoising, double threshold mapping is adopted, and finally, a dual threshold mapping infrared image processing method based on adaptive wavelet denoising algorithm based on binary wavelet transform is formed.

Simulation results show that the method proposed in this paper has a better suppression of noise and improves the image quality to a certain extent.

\section{Related work}

Nowadays, the denoising method based on wavelet transform has become an important branch of image denoising and restoration. Among the many image denoising methods based on wavelet transform, the wavelet threshold denoising method proposed by Donoho [6] and others has been widely used because of its simple principle, easy implementation, and remarkable denoising effect. But its inherent shortcomings, such as the discontinuity of hard threshold function, the constant deviation caused by soft threshold function [7-10], and the lack of scale adaptability, also limit its further development. Later, many scholars and researchers have studied it deeply from the point of view of constructing new threshold function and searching for optimal threshold and proposed many adaptive image denoising methods. Hong and Yang [11] have compared the signal-to-noise ratio (SNR) and root mean square error (RMSE) according to the shortcomings of soft threshold function and hard threshold function in the 
traditional wavelet threshold denoising algorithm and constructed a new threshold function to denoise the signal. To a large extent, the shortcomings of poor continuity and inherent deviation of these two functions were overcome in practical processing, which made the noise processing more effective and superior. Juncheng and Qiang [12] applied the translation invariant to the second-generation wavelet transform based on the existing threshold denoising method of the second-generation wavelet transform and realized the more rapid and effective denoising of seismic signal and have achieved good denoising effect in the trial calculation of analog data and actual data. Hong [13] improved the classical wavelet threshold denoising algorithm and proposed a new threshold denoising method of mine video monitoring image in the wavelet transform domain. The image was decomposed by three-level wavelet transform, the low-frequency decomposition coefficients were filtered by Wiener filter, and the high-frequency decomposition coefficients were denoised by improved wavelet $I$ value denoising algorithm. Experiments showed that it can effectively reduce noise and get an effective and complete image. Xiaofei and Xiaohui [14] combined the advantages of typical wavelet threshold function, integrated some improved methods, and proposed an improved threshold function for its shortcomings. This function is not only continuous at the threshold; the coefficient is a symptotic original coefficient of the wavelet estimation, but also has differentiability, and it is easy to implement adaptive learning of the gradient algorithm. Fengbo, Changgeng, and Hongqiu [15] proposed an improved threshold algorithm based on lifting wavelet transform. First of all, the advantages of the wavelet transform, multiresolution, and diversity of wavelet bases were exploited. On the other hand, a new threshold function was proposed to improve the signal-to-noise ratio and reduce the RMSE. Denoising with the improved threshold function is superior to the commonly used threshold function in both visual effects and mean square error and peak signal-to-noise ratio performance analysis. Xiaoyan and Abdukirimturki [16] combined the advantages of typical wavelet threshold function with some improved methods and proposed an improved new threshold function. The experimental results show that the denoised image is better than the traditional soft and hard thresholding and the existing thresholding in terms of visual effect, mean square deviation, and peak signal-to-noise ratio. These methods not only open up a broad prospect for the full advantage of the wavelet threshold denoising method but also provide a basis for further exploration of adaptive denoising methods. However, in general, these studies are almost all based on the orthogonal wavelet transform, using the Mallat algorithm. Mallat algorithm [17] can achieve satisfactory speed, but due to the lack of translation invariance, the reconstruction accuracy after image denoising is not high, and the Gibbs phenomenon is easy to appear in the denoised image and cannot meet the requirements of human vision. The fast algorithm based on the binary wavelet transform [18] -à trous algorithm [19] — not only has the invariance of translation but also makes the representation of the image in the domain of binary wavelet transform very redundant, and the disturbance of partial coefficients will not lead to the serious distortion of the reconstructed image. In addition, for the Gibbs visual distortion in a denoised image caused by the wavelet threshold denoising method using orthogonal wavelet transform, many researchers have made improvements such as literature [20-23]. The results show that translation invariance is an important property of effectively suppressing Gibbs phenomenon and improving denoising effect. These conclusions provide a basis for the research of adaptive threshold image denoising based on binary wavelet transform.

\subsection{Infrared image noise analysis}

\subsubsection{The meaning and classification of image noise}

Image noise is generally defined from two aspects of human perception and mathematics. From the mathematical point of view, the image information can be regarded as a spatial function $f$, and image noise is the factor that degrades the information expressed by this function, that is, under the influence of noise, the image is degraded to $f_{n}$. Image noise can be divided into different categories according to different methods. From the mathematical point of view, the image can be divided into additive noise and multiplicative noise according to the way in which the image is degraded. These two relations can be expressed by the following formula:

$$
\begin{aligned}
& f_{n}=f+n \\
& f_{n}=f \cdot n
\end{aligned}
$$

where $n$ represents noise, formula (1) represents additive noise, and (2) represents multiplicative noise.

If it is divided according to the physical factors of noise generation, it can be classified into electronic noise, photoelectron noise, photosensitive particle noise, speckle noise, and so on.

\subsubsection{Measurement of image denoising effect}

Because of the randomness of noise generation itself, the noise contained in an image can only be described by a statistical method, that is to say, the noise generation is regarded as a random process. The probability density function of this random process is used to describe the overall behavior of noise. But in many cases, the probability density function of noise distribution is very difficult to obtain, or it is difficult to express by 
some mathematical function. In this case, we usually study some digital characteristics of noise distribution, such as the mean $m$, variance $\sigma$, correlation coefficient $\rho$, and so on. This paper describes the following indicators for measuring the quality of image denoising:

1. Normalized mean square error (NMSE)

$$
\mathrm{NMSE}=\frac{\sum_{m} \sum_{n}\left[f(m, n)-f^{\prime}(m, n)\right]^{2}}{\sum_{m} \sum_{n} f(m, n)^{2}}
$$

where $f(m, n)$ is the original image, and $\dot{f}(m, n)$ represents the restored image after processing.

2. Standard deviation $s$

$$
s=\sqrt{\frac{\sum_{i=1}^{M} \sum_{j=1}^{N}(f(i, j)-\bar{f})^{2}}{M N}}
$$

where $f(i, j)$ is an image, $\bar{f}=\frac{\sum_{i=1}^{M} \sum_{j=1}^{N} f(i, j)}{M N}$, and $M$ and $N$ are the total number of rows and columns of the image matrix.

3. The ratio between signal and mean squared error (F/MSE)

$$
\mathrm{F} / \mathrm{MSE}=10 \lg \frac{\sum_{m=1}^{M} \sum_{n=1}^{N}[f(m, n)]^{2}}{\sum_{m=1}^{M} \sum_{n=1}^{N}\left[f^{\prime}(m, n)-f(m, n)\right]^{2}}(\mathrm{~dB})
$$

4. Image relative signal to noise ratio

$$
\mathrm{SNR}=\frac{\bar{f}}{s}
$$

5. Smoothness: In most cases, the image after denoising should be at least as smooth as the original image.

6. The variance estimation of image and original image after similarity denoising should be the least variance in the worst case (minmax estimator).

\subsubsection{Noise characteristic analysis of infrared image}

Speckle noise [24] exists extensively in the imaging process of infrared images. The formation of speckle noise is mainly due to the interaction of infrared waves in the imaging process. Besides, it is also closely related to the roughness of the imaging tissue surface. From the visual point of view, the noise in the image presents a speckle distribution and according to the density of speckles can be divided into three types of noise.

From the nature of noise analysis, strictly speaking, speckle noise contains both the components of multiplicative noise and the components of additive noise, which can be expressed by Eq. (7):

$$
f_{n}=f \cdot n_{m}+n_{a}
$$

However, in most cases, the influence of additive noise on the image is much smaller than that of the multiplicative noise, so the additive noise can be ignored. Finally, the speckle noise is considered to be a multiplicative noise.

\subsection{Binary wavelet theory}

In the Mallat algorithm, due to the downsampling operation, the singular points of the image edges are easily lost, resulting in Gibbs phenomenon at the edges of the reconstructed images after denoising, resulting in image edge distortion or even blur. In order to overcome this drawback, wavelet transform with translation invariance needs to be considered. The binary wavelet transform only discretizes the scale factor in the continuous wavelet transform and keeps the translation factor continuously changing, thus effectively maintaining the translation invariance. This feature of the binary wavelet transform, coupled with the fast decomposition and reconstruction algorithm, à trous algorithm, has certain advantages in the fields of image denoising and image feature extraction.

It should be noted that the à trous algorithm mentioned in this paper is a modified version of the à trous algorithm form described in the Mallat book, and the algorithm is different from the proof in the Mallat book.

Assuming that the sample value $a_{0, k}$ of the input discrete signal is the local average of $f$ in the domain of $t=k$, it can be written as:

$$
a_{0, k}=<f(t), \varphi(t-k)>
$$

where $\varphi$ and $\tilde{\varphi}$ are the two scale functions generated by the infinite cascade calculation of filter $(h, g)$ and its dual filter $(\tilde{h}, \tilde{g})$, which can be expressed as: 


$$
\begin{aligned}
& \phi(t)=\sqrt{2} \sum_{k} h_{k} \varphi(2 t-k) \\
& \tilde{\phi}(t)=\sqrt{2} \sum_{k}^{k} \tilde{h}_{k} \tilde{\varphi}(2 t-k)
\end{aligned}
$$

For any $j \geq 0$, remember:

$$
a_{j, k}=<f(t), \varphi_{2^{j}}(t-k)>
$$

where $\varphi_{2^{j}}(t)=\frac{1}{\sqrt{2^{j}}} \varphi\left(\frac{t}{2^{j}}\right)$.

The binary wavelet coefficients are calculated as follows:

$$
d_{j, k}=W f\left(2^{j}, k\right)=<f(t), \psi_{2^{j}}(t-k)>
$$

where $\psi_{2^{j}}(t)=\frac{1}{\sqrt{2^{j}}} \psi\left(\frac{t}{2^{j}}\right)$ and $\psi$ and $\tilde{\psi}$ are the wavelet functions generated by the infinite cascade calculation of the filter $(h, g)$ and its dual filter $(\tilde{h}, \tilde{g})$, which can be expressed as:

$$
\begin{aligned}
\psi(t) & =\sqrt{2} \sum_{k} g_{k} \varphi(2 t-k) \\
\tilde{\psi}(t) & =\sqrt{2} \sum_{k} \tilde{g}_{k} \tilde{\varphi}(2 t-k)
\end{aligned}
$$

For the à trous algorithm, the binary wavelet decomposition algorithm is expressed as:

$$
\begin{aligned}
& a_{j+1, k}=\sum_{n} h_{n} a_{j, k+2^{j} n}, j=0,1, \cdots, \\
& d_{j+1, k}=\sum_{n} g_{n} a_{j, k+2^{j} n}, j=0,1, \cdots .
\end{aligned}
$$

The binary wavelet reconstruction algorithm is expressed as:

$$
\begin{aligned}
a_{j, k} & =\frac{1}{2}\left(\sum_{n} \tilde{h}_{n} a_{j+1, k-2^{j} n}+\sum_{n} \tilde{g}_{n} d_{j+1, k-2^{j} n}\right), j \\
& =0,1, \cdots
\end{aligned}
$$

The proofs of formulas (13) and (14) are given below. For formula (13), the following can be obtained from the definition of $a_{j, k}$ and the relationship between two scales.

$$
\begin{aligned}
a_{j+1, k} & =<f(t), \varphi_{2^{j+1}}(t-k)>=\int_{R} f(t) \frac{1}{\sqrt{2^{j+1}}} \varphi^{*}\left(\frac{t-k}{2^{j+1}}\right) d t \\
& =\sum_{n} h_{n} a_{j, k+2^{j} n}, \quad j=0,1, \cdots .
\end{aligned}
$$

In the same way, there are:

$$
\begin{aligned}
d_{j+1, k} & =<f(t), \psi_{2^{j+1}}(t-k)>=\int_{R} f(t) \frac{1}{\sqrt{2^{j+1}}} \psi^{*}\left(\frac{t-k}{2^{j+1}}\right) d t \\
& =\sum_{n} g_{n} a_{j, k+2^{j} n}, \quad j=0,1, \cdots .
\end{aligned}
$$

For formula (15), from the dual-scale relationship $\widehat{\tilde{\varphi}}(2 w)=\frac{1}{\sqrt{2}} \widehat{\tilde{h}}(w) \widehat{\tilde{\varphi}}(w), \quad \widehat{\tilde{\psi}}(2 w)=\frac{1}{\sqrt{2}} \widehat{\tilde{g}}(w) \widehat{\tilde{\varphi}}(w)$ and the binary condition $\hat{\psi}^{*}(w) \widehat{\tilde{\psi}}(w)+\hat{\varphi}^{*}(w) \widehat{\tilde{\varphi}}(w)=\hat{\varphi}^{*}(w / 2) \widehat{\tilde{\varphi}}$ $(w / 2)$, we get:

$$
\sqrt{2} \hat{\varphi}\left({ }^{w} / 2\right)=\widehat{\widetilde{h}}^{*}(w / 2) \hat{\varphi}(w)+\widehat{\widetilde{g}}^{*}(w / 2) \hat{\psi}(w)
$$

Time domain is expressed as:

$$
2 \sqrt{2} \varphi(2 t)=\sum_{k} \tilde{h}_{2 k} \varphi(t+k)+\sum_{k} \tilde{g}_{2 k} \psi(t+k)
$$

At this time, for formula (14), there is:

$$
\begin{aligned}
a_{j, k} & =<f(t), \varphi_{2^{j}}(t-k)>=\int_{R} f(t) \frac{1}{\sqrt{2^{j}}} \varphi^{*}\left(\frac{t-k}{2^{j}}\right) d t \\
& =\frac{1}{2}\left(\sum_{k} \tilde{h}_{k} a_{j+1, k-2^{j} n}+\sum_{k} \tilde{g}_{k} d_{j+1, k-2^{j} n}\right), j=0,1, \cdots .
\end{aligned}
$$

The above theorem describes the à trous algorithm of the one-dimensional signal. The structure of this algorithm is shown in Fig. 1. For the two-dimensional image signal, the one-dimensional à trous algorithm is applied along the rows and columns of the image to realize image decomposition and reconstruction.

Although the à trous algorithm has the same algorithm structure as the Mallat algorithm, there is a substantial difference: the à trous algorithm does not perform the downsampling operation, which not only preserves the translation invariance well but also the data length under each scale is the same as the original data length. Compared with the data of the Mallat algorithm, there is a great redundancy, which is convenient for spectrum analysis of details and profiles at each scale.

\section{Proposed method}

\subsection{Adaptive threshold denoising analysis of wavelet} transform

3.1.1 The basic principle of image denoising based on wavelet transform

The idea of image denoising based on wavelet transform can be roughly divided into two kinds: one is that 


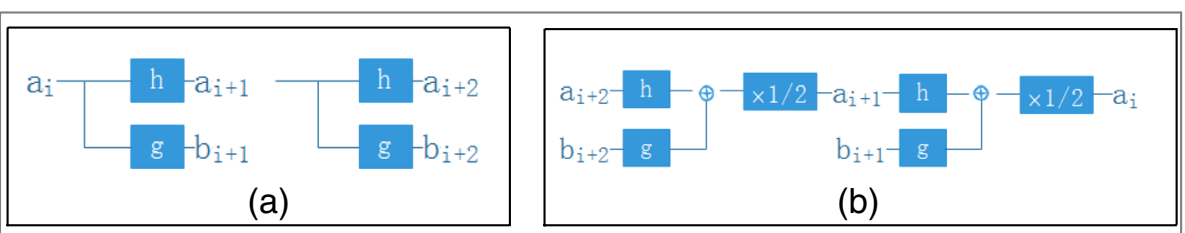

Fig. 1 a à trous decomposition algorithm. $\mathbf{b}$ à trous reconstruction algorithm

the image signal and noise signal show different laws in different resolutions after wavelet transform. The threshold can be set at different resolution levels, and the wavelet coefficients can be adjusted to achieve the purpose of denoising. The other is to combine wavelet transform with traditional image denoising algorithm to improve the performance of the original image denoising algorithm by utilizing the characteristics of wavelet transform multiresolution and time-frequency localization. The general steps of wavelet transform for image denoising are as follows:

1. Two-dimensional discrete wavelet transform is used to get the wavelet coefficients at different resolutions.

2. The wavelet coefficients are analyzed and adjusted accordingly or processed according to some traditional methods.

3. Wavelet transform is used to get the processed image.

From the above description, the key of image denoising using wavelet transform is the selection of wavelet basis function and the analysis and correction of wavelet coefficients. Based on the threshold processing method of wavelet coefficients, the main idea of this method is to divide wavelet coefficients into noise-contaminated points and information points and then filter wavelet coefficients representing noise points. The remaining wavelet coefficients are used to reconstruct the image to get the denoising results.

\subsubsection{Research on threshold processing of wavelet coefficient}

From the energy point of view, the energy of the noise on the wavelet domain is distributed over all wavelet coefficients, while the signal energy is concentrated on only a small fraction of the wavelet coefficients. Therefore, the wavelet coefficients can be divided into two types. The first type of wavelet coefficients is only obtained by noise transformation. The wavelet coefficients have small amplitude and a large number. The second type of wavelet coefficients is derived from the signal and contains the influence of noise. These wavelet coefficients have large amplitudes and a small number. In this way, the threshold can be set by the difference in the amplitude of the wavelet coefficients, and a denoising method can be constructed. A wavelet coefficient larger than this threshold is considered to belong to the second type of coefficient, that is, a transformation result containing both signals and noise, which may retain a simple reservation or a subsequent operation. And a wavelet coefficient smaller than the threshold is considered to be the first type of wavelet coefficient, that is, it is completely transformed by noise, and the removal of these coefficients achieves the purpose of denoising. At the same time, the image details can be better preserved by retaining most of the wavelet coefficients containing the signal. The wavelet threshold denoising method can be described by the following three-step strategy:

1. Calculate the orthogonal wavelet transform of noisy images

Select the appropriate wavelet base and wavelet decomposition layer number $J$, and use the Mallat decomposition algorithm to perform the $J$-layer wavelet decomposition of the noisy image to obtain the corresponding wavelet decomposition coefficient.

2. Threshold quantization of the decomposed highfrequency coefficients

For each layer from 1 to $J$, an appropriate threshold and an appropriate threshold function are selected to threshold quantize the decomposed high-frequency coefficients to obtain an estimated wavelet coefficient.

\section{Perform inverse wavelet transform}

According to the low-frequency coefficient (scale coefficient) of the Jth layer after image wavelet decomposition and the high-frequency coefficients (wavelet coefficients) of each layer after threshold quantization, the Mallat reconstruction algorithm is used for wavelet reconstruction to obtain the denoised image.

It can be seen from the above description that the selection of the threshold value, the threshold function, the selection of the wavelet basis, and the determination of the decomposition layer are the key factors in the wavelet threshold denoising method. 


\subsubsection{Adaptive threshold function selection}

In the wavelet threshold technique, the choice of threshold is a key factor in determining the performance of the algorithm. On the one hand, it needs to be large enough to remove as much noise as possible, and on the other hand, it needs to be small enough to retain as much signal energy as possible. In many practical applications, it is difficult to meet both of these requirements by relying on a single global uniform threshold. Since the wavelet transform is a multiresolution analysis, the selection of the threshold can be considered on each layer. That is, the local feature of the image is used as a parameter for threshold selection so that the threshold can be changed as the specific characteristics of the image change. In this paper, an adaptive threshold function is designed. Compared with the hard threshold and soft threshold function, it has better superiority and flexibility. It can realize different processing of wavelet coefficients and complete adaptive denoising of images.

Although the hard threshold and soft threshold methods have been widely used in practice, they have achieved good results, but the method itself has some potential defects:

1. The hard threshold function uses the threshold as the boundary between the signal and the noise. The coefficient smaller than the threshold is set to zero, and the coefficient larger than the threshold is directly retained. This method preserves most of the details of the image well, which brings great convenience to the processing of the image. But it does not match the interference of the noise signal in the wavelet coefficient larger than the threshold in the actual situation, and the lack of continuity at the threshold makes the estimated signal produce additional oscillations, resulting in visual distortion such as pseudo-Gibbs phenomenon in the reconstructed image.

2. The continuity of the soft threshold function at the threshold can effectively overcome the pseudo-Gibbs phenomenon caused by the hard threshold function, but the constant deviation between the estimated wavelet coefficients and the wavelet coefficients of the soft threshold function has a direct impact on the approximation between the reconstructed image and the real image. The result of denoising by soft threshold method is relatively smooth, and it is easy to cause blurred image edges.

In this paper, based on the advantages and disadvantages of the soft and hard threshold functions, an adaptive threshold function is constructed in combination with the literature [25-28]. This function can be regarded as a generalization of soft and hard threshold functions. It is based on the nonlinear hyperbolic tangent function of $\mathrm{BP}$ neural network; the translation of the function is adjusted by introducing the threshold $\delta$. And based on parameter $m$, the difference between the estimated wavelet coefficient and the wavelet coefficient can be adjusted. The expression is as follows:

$$
\hat{W}_{i, j}=\left\{\begin{array}{ll}
\operatorname{sgn}\left(w_{i, j}\right)\left(\left|w_{i, j}\right|-m \delta \frac{1-e^{-\left(\left|w_{i, j}\right|-\delta\right)}}{1+e^{-\left(\left|w_{i, j}\right|-\delta\right)}}\right), & \left|w_{i, j}\right| \geq \delta \\
0, & \left|w_{i, j}\right|<\delta
\end{array}, m \geq 0\right.
$$

Looking at the above adaptive threshold function, it can be seen that when parameter $m=0$, this function is a hard threshold function; when $m=1$, and $w_{i, j} \rightarrow \infty$, the function is a soft threshold function; when $\mid w_{i}$, $j|\rightarrow \infty,| \hat{w}_{i, j}-w_{i, j} \mid \rightarrow m \delta$, that is, as $\left|w_{i, j}\right|$ increases, the absolute value of the deviation of the estimated wavelet coefficient $\hat{w}_{i, j}$ and the wavelet coefficient $w_{i, j}$ gradually approaches $m \delta$. Since $m$ is a parameter, the absolute value of the deviation can be controlled by adjusting the size of $m$, which effectively improves the constant deviation between the estimated wavelet coefficient and the wavelet coefficient generated in the soft threshold function. Compared with the soft and hard thresholding function, the adaptive thresholding function proposed in this paper is a more superior and flexible choice. It can realize the different processing of wavelet coefficients and complete the adaptive denoising of images only by properly determining the size of $\mathrm{m}$ according to different images.

\subsection{Infrared image processing based on binary wavelet adaptive threshold denoising}

It can be seen from Section 2.1 that the infrared image noise is mainly due to the coherence of infrared light waves, including both multiplicative noise and additive noise. But in most cases, the effect of additive noise on the image is much less than that of multiplicative noise. However, most of the current wavelet denoising methods are designed for additive noise interference, which obviously cannot meet the needs of infrared image processing in this subject. Therefore, a binary wavelet adaptive threshold denoising scheme is designed for speckle noise in the infrared image.

Considering that speckle noise is dominated by multiplicative noise, the multiplicative noise is transformed into additive noise by taking the logarithm of infrared image, then the binary wavelet adaptive threshold denoising algorithm is used to process the image, and then the exponential restoration of the processing results can be achieved. According to the calculation, the pixel values of the infrared image used in the experiment are all positive, so the logarithm scheme can be adopted. 
In summary, the algorithm is described as follows:

1. Assuming that the additive noise in speckle noise is ignored, formula (8) can be rewritten as:

$$
f_{n}=f \cdot n_{m}
$$

2. Take the logarithm of 4 as the base on both sides of formula (24), we can get:

$$
\log _{4} f_{n}=\log _{4} f+\log _{4} n_{m}
$$

In fact, in order to avoid taking the logarithm of 0 as the base when implementing the algorithm, special order $f_{n}=f_{n}+0.0000001$

3. The infrared image $\log _{4} f_{n}$ performs three-layer binary wavelet decomposition: select appropriate wavelet base for $\log _{4} f_{n}$, and apply à trous decomposition algorithm for $J$-layer decomposition to obtain a low-frequency (scale) coefficient $a_{j, m, n}$ and a set of high-frequency coefficients $d_{j, m, n}^{i}(i=1,2,3)$, where $i=1,2,3$ respectively indicate horizontal, vertical, and diagonal directions.

4. Threshold quantification of high-frequency coefficients: First, the high-frequency coefficient $d_{j, m, n}^{i}(i=1,2,3)$ is rewritten as a one-dimensional sequence $d_{j, k}^{i}(i=1,2$, $3)$, and then the estimation coefficient $\hat{d}_{j, k}^{i}(i=1,2,3)$ is obtained by double threshold processing of $d_{j, k}^{i}(i=1$, 2,3 ) with the adaptive threshold function given in this paper. The expression of the adaptive threshold function is rewritten as follows:

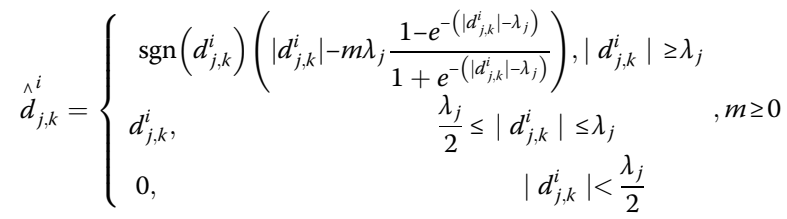

The upper $\operatorname{sgn}(\cdot)$ is a symbolic function, $\lambda_{j}=\sigma$ $\sqrt{2 \ln (M N)} / \ln (j+1)$ is a scale adaptive threshold,

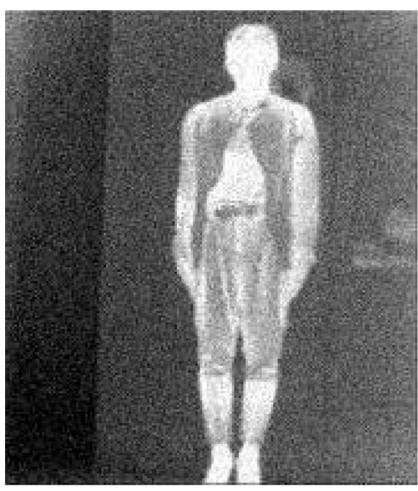

a The original image

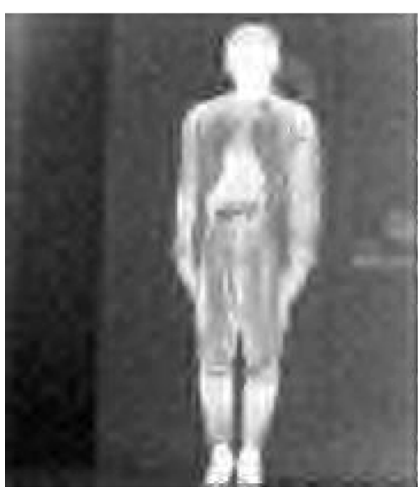

C Hard threshold function denoising

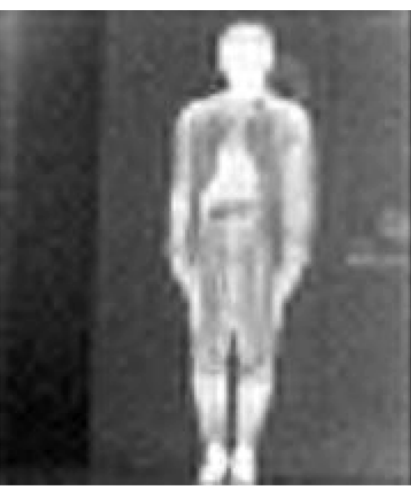

b Soft threshold function denoising

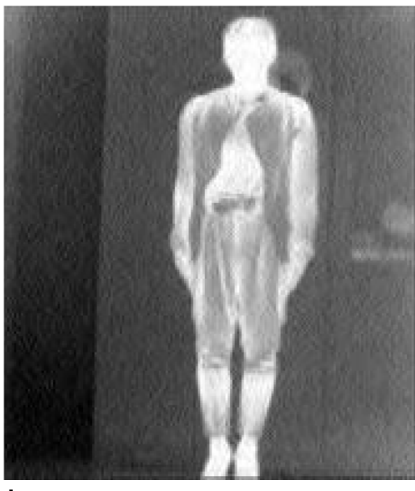

d Adaptive function denoising

Fig. 2 Results graph of denoising for different threshold functions of males. a The original image. $\mathbf{b}$ Soft threshold function denoising. $\mathbf{c}$ Hard threshold function denoising. $\mathbf{d}$ Adaptive function denoising 
Table 1 Comparison of different threshold function denoising data

\begin{tabular}{|c|c|c|c|c|c|c|}
\hline \multirow{2}{*}{$\begin{array}{l}\text { Gender } \\
\text { Index }\end{array}$} & \multicolumn{3}{|l|}{ Male } & \multicolumn{3}{|l|}{ Female } \\
\hline & Standard deviation $s$ & $\begin{array}{l}\text { SNR or F/ } \\
\text { MSE(dB) }\end{array}$ & NMSE & Standard deviation $s$ & $\begin{array}{l}\text { SNR or F/ } \\
\text { MSE(dB) }\end{array}$ & NMSE \\
\hline Original infrared image & 82.12 & 12.01 & / & 82.25 & 12.13 & / \\
\hline Soft threshold denoising image & 78.25 & 13.03 & $9.1367 e-005$ & 77.53 & 12.96 & $9.0304 \mathrm{e}-005$ \\
\hline Hard threshold denoising image & 77.57 & 12.91 & $8.9812 \mathrm{e}-005$ & 77.11 & 13.23 & $8.8911 \mathrm{e}-005$ \\
\hline The denoising image of algorithm in this paper & 21.07 & 15.65 & $6.5287 \mathrm{e}-005$ & 20.99 & 15.47 & $6.7044 \mathrm{e}-005$ \\
\hline
\end{tabular}

$M$ and $N$ are the rows and columns of image pixels, $j$ is the decomposition scale, and $\sigma$ is the noise standard deviation, expressed as: $\sigma^{2}=\operatorname{median}\left\{d_{j, m, n}^{i}\right\} / 0.674$ $5, i=1,2,3,1 \leq j \leq J$.

5. Reconstruction of infrared image $\log _{4} f_{n}$ : Write the estimated coefficient $\hat{d}_{j, k}^{i}(i=1,2,3)$ into a two-dimensional form $\hat{d}_{j, m, n}^{i}(i=1,2,3)$; combined with the low-frequency coefficient $a_{j}$ $m, n$, the image is reconstructed by the à trous reconstruction algorithm.

6. The image obtained in the previous step is subjected to a power of 4 to obtain a denoised image.

\section{Results and discussion}

The simulated image is uniformly imaged by an infrared image of $182 \times 208$ pixels, which is taken by a medical infrared camera of Chongqing Weilian Technology Limited. The selected wavelet functions are sym4 wavelets with good symmetry and suitable for image denoising.

Firstly, the performance of the adaptive threshold function selected in this paper is analyzed, and the traditional soft and hard threshold functions are used as a comparison. The results of infrared image processing are shown in
Fig. 2. Figure $2 \mathrm{a}$ is the original image, Fig. $2 \mathrm{~b}$ is the result of soft threshold function denoising, Fig. $2 \mathrm{c}$ is the result of hard threshold function denoising, and Fig. $2 \mathrm{~d}$ is the result of adaptive threshold function denoising designed in this paper. As can be seen in Fig. 2, the resulting image obtained by soft and hard threshold function denoising still contains a lot of noise compared with the original infrared image, and the visual effect is not much improved compared with the original infrared image. The overall effect of the image obtained by adaptive threshold denoising in this paper is relatively smooth and clear, so it can be said that the adaptive threshold function proposed in this paper is better for infrared image denoising.

In order to better compare the performance of adaptive threshold function processing proposed in this paper, formula (6) is used to calculate the signal-tonoise ratio of the original image, and formula (5) is used to calculate the signal-to-mean square error ratio of image denoising. The comparison results are shown in Table 1.

In order to better show the denoising effect of soft and hard thresholding functions and adaptive thresholding functions of this algorithm, the standard deviation of denoising of different thresholding functions for men
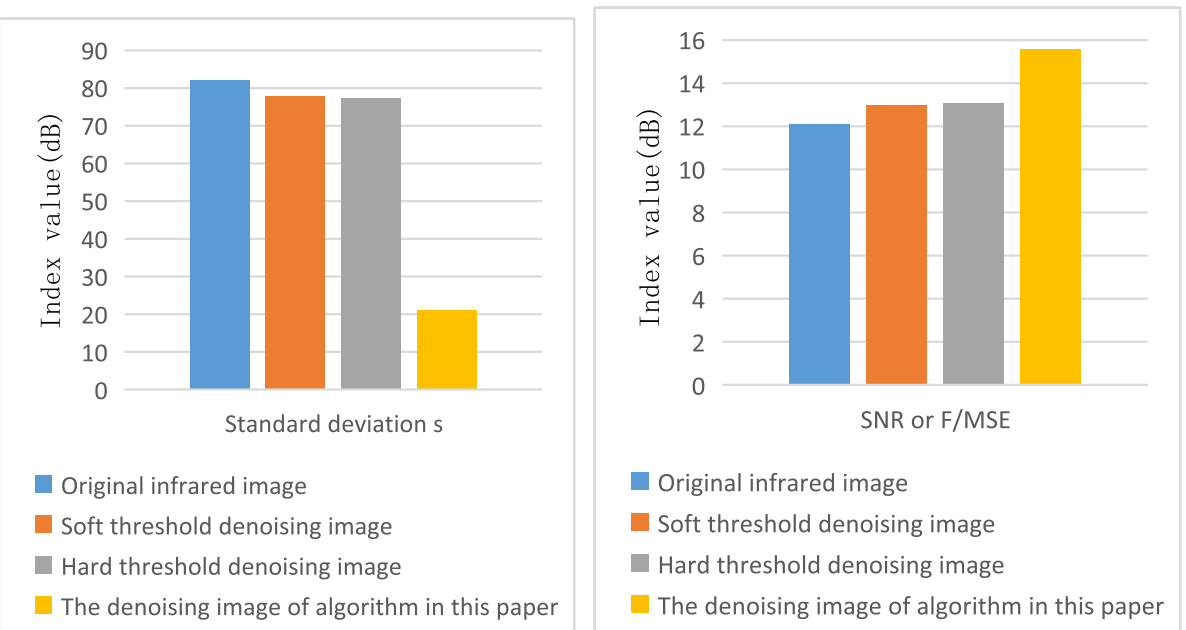

Fig. 3 Results graph of denoising for different threshold functions of females 


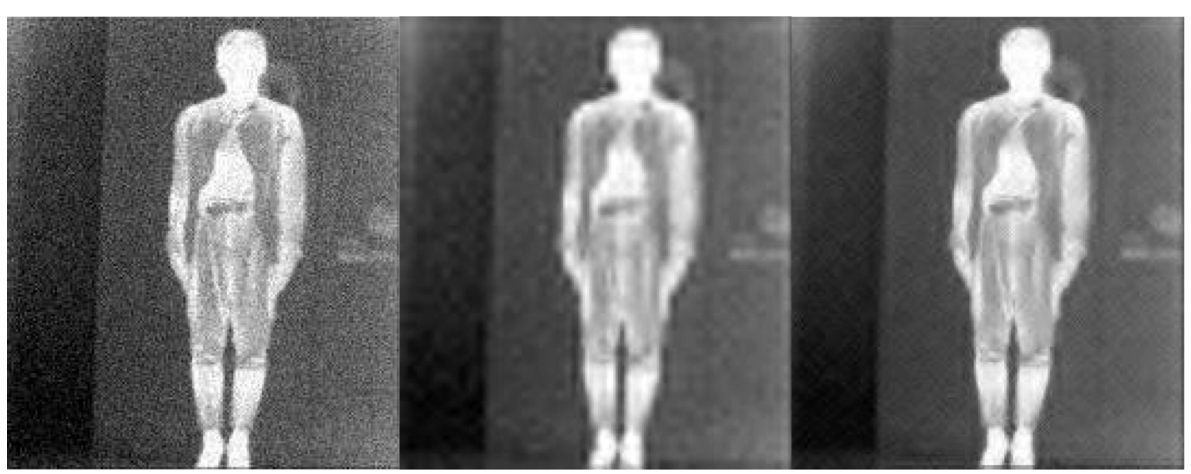

(a)Original Image (b)Traditional wavelet threshold denoising results (c)Denoising result of this algorithm

Fig. 4 Simulation results of males. a Original image. b Traditional wavelength threshold denoising results. $\mathbf{c}$ Denoising result of this algorithm

and women in Table 1 and SNR or F/MSE is averaged, and the histogram is drawn as shown in Fig. 3. Combining Fig. 3 and Table 1, we can see that the soft and hard threshold functions can denoise the image and raise the standard deviation by about 5 and the SNR or F/MSE by about $1 \mathrm{~dB}$. The adaptive threshold function proposed in this paper can denoise the image and raise the standard deviation by about 60 and the SNR or F/MSE by about $3 \mathrm{~dB}$.

In order to validate the performance of the infrared image denoising algorithm designed in this paper, the traditional wavelet threshold denoising algorithm and the algorithm designed in this paper are used to denoise the infrared image. The results are shown in Fig. 4, in which Fig. $4 \mathrm{a}$ is the original image, Fig. $4 \mathrm{~b}$ is the denoising result of the traditional wavelet threshold denoising algorithm, and Fig. 4c is the denoising result of the algorithm designed in this paper. In Fig. 4, we can see that the traditional wavelet threshold denoising algorithm can effectively improve the performance of infrared image, but the image denoised by this algorithm retains the edge features of the image and effectively improves the Gibbs phenomenon near the edge and the image details are clearer and the visual effect is obviously better than the traditional wavelet denoising method.

Similarly, Table 2 gives the performance data of the traditional wavelet threshold method compared with the algorithm in this paper. In order to better show the denoising effect of the traditional wavelet threshold algorithm and the algorithm in this paper, the standard deviation of different threshold functions for men and women in Table 2 and SNR or F/MSE is averaged, and the histogram is drawn as shown in Fig. 5. Combining Table 2 and Fig. 5, we can see that the traditional wavelet threshold algorithm can improve the standard deviation by about 5 and the SNR or F/MSE by about $1 \mathrm{~dB}$, while the image denoising by this algorithm can improve the standard deviation by about 67 and the SNR or F/MSE by about $6 \mathrm{~dB}$.

In summary, experiments show that the proposed method for infrared image denoising has better performance than the traditional wavelet threshold denoising algorithm and can effectively suppress the Gibbs visual distortion caused by the lack of translation invariance of orthogonal wavelet transform.

\section{Conclusions}

With the implementation of the Digital Earth Project and the development of infrared technology, infrared image processing has become the main factor restricting the development of infrared technology. Therefore, the key to the breakthrough development of infrared image processing is to adopt new and effective mathematical analysis methods instead of traditional analysis methods. Based on the traditional wavelet threshold infrared image denoising method, this paper fuses the image adaptive threshold denoising algorithm and carries on the double threshold mapping processing to the infrared image. Firstly, on the basis of traditional wavelet threshold processing of

Table 2 Data comparison of image denoising

\begin{tabular}{|c|c|c|c|c|c|c|}
\hline \multirow{2}{*}{$\begin{array}{l}\text { Gender } \\
\text { Index }\end{array}$} & \multicolumn{3}{|l|}{ Male } & \multicolumn{3}{|l|}{ Female } \\
\hline & Standard deviation $s$ & $\begin{array}{l}\text { SNR or F/ } \\
\text { MSE(dB) }\end{array}$ & NMSE & Standard deviation $s$ & $\begin{array}{l}\text { SNR or F/ } \\
\text { MSE(dB) }\end{array}$ & NMSE \\
\hline Original infrared image & 82.12 & 12.01 & l & 82.25 & 12.13 & / \\
\hline Traditional wavelet threshold denoising image & 78.25 & 13.03 & $9.1367 \mathrm{e}-005$ & 77.53 & 12.96 & $9.0304 \mathrm{e}-005$ \\
\hline Image denoising based on this algorithm & 15.22 & 18.53 & $4.1217 e-005$ & 15.78 & 18.62 & $4.2077 \mathrm{e}-005$ \\
\hline
\end{tabular}



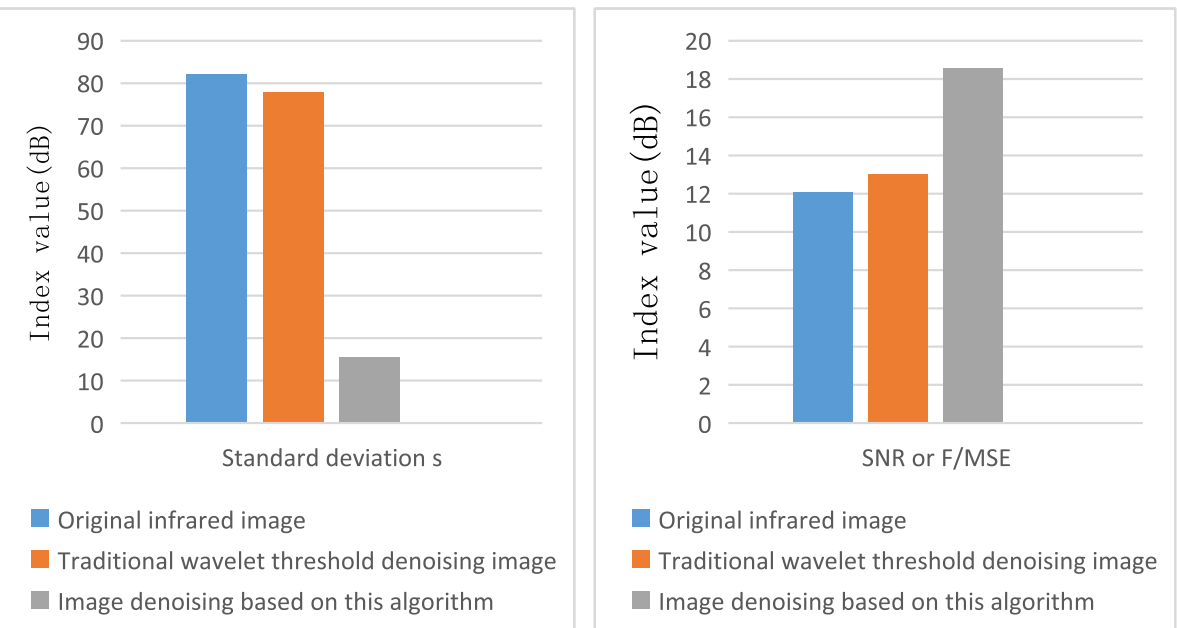

Fig. 5 Simulation results of females

infrared images, the advantages and disadvantages of soft and hard threshold functions are analyzed in depth, and an adaptive threshold function with adjustable parameters is constructed, and a double threshold mapping method is used to process infrared images. Secondly, in order to suppress the Gibbs visual distortion caused by the lack of translation invariance of orthogonal wavelet transform, a binary wavelet transform with translation invariance is introduced. Finally, a dual threshold mapping infrared image processing method based on the adaptive threshold denoising algorithm of binary wavelet transform is formed. Simulation results demonstrate the effectiveness and superiority of the proposed method.

\section{Abbreviations}

F/MSE: The ratio between signal and mean squared error; NMSE: Normalized mean square error

\section{Acknowledgements}

The author thanks the editor and anonymous reviewers for their helpful comments and valuable suggestions.

\begin{abstract}
About the authors
Jilin University at Changchun, No.2699 Qianjin Rd. Chaoyang District, Changchun, Jilin.

Binbin Yu was born in Jilin City, Jilin Province. P.R. China, in 1984. He received the Master degree from Northeast Normal University, P.R. China. Now, he is a Ph.D. candidate, College of Computer Science and Technology, Jilin University, He works in Computer Science and Technology College, Beihua University. His research interests include information fusion of Internet of things and so on.
\end{abstract}

\section{Funding}

No funding.

\section{Availability of data and materials}

Please contact author for data requests.

\section{Authors' contributions}

BY did everything in this paper. The author read and approved the final manuscript.

\section{Competing interests}

The author declares that he has no competing interests.

\section{Publisher's Note}

Springer Nature remains neutral with regard to jurisdictional claims in published maps and institutional affiliations.

Received: 2 September 2018 Accepted: 21 December 2018

Published online: 07 January 2019

\section{Referencess}

1. M. Wang, F. Yangyu, C. Baoguo, et al., Realization of adaptive nonuniformity correction of infrared image based on SOPC. Infr. Laser Eng. 46(6), 628001 (2017)

2. X. Li, Y. Jiang, M. Chen, et al., Research on iris image encryption based on deep learning. EURASIP J. Image Video Proc. 2018(1), 126 (2018)

3. Hu L, Zhang J, Zhang L, Real-Time Infrared Target Detection Method Based on Human Vision System. Journal of Northwestern Polytechnical University. 35(5), 910-914 (2017)

4. O. Prakash, J. Gwak, M. Khare, et al., Human detection in complex real scenes based on combination of biorthogonal wavelet transform and Zernike moments. Optik 157, 1267-1281 (2018)

5. Z. Zhenfeng, W. Huan, B. Tan, An improved wavelet threshold denoising method. Study Opt. Commun. 2(206), 75-78 (2018)

6. D.L. Donoho, De-noising by soft-thresholding. IEEE Trans. Inf. Theory 41(3), 613-627 (2002)

7. S. Duan, X. Hu, L. Wang, S. Gao, C. Li, Hybrid memristor/RTD structure-based cellular neural networks with applications in image processing. Neural Comput. Applic. 25(2), 291-296 (2014)

8. W.U. Anquan, C. Shen, J. Xiao, et al., Wavelet denoising based on an asymptotic semisoft thresholding function. Chin. J. Electron Devices (2017)

9. D. Han, Research on improved threshold de-noising method of gyro signal. Aeronaut. Manuf. Technol. (2016)

10. N.K. Alham, M. Li, Y. Liu, Parallelizing multiclass support vector machines for scalable image annotation. Neural Comput. Applic. 24(2), 367-381 (2014)

11. J. Hong, S. Yang, Denoising method based on improved wavelet threshold function. Laser Infrared 1(46), 119-122 (2016)

12. L. Juncheng, Z. Qiang, Application of improved threshold method on second-generation wavelet transform denoising. Glob. Geol. 36(2), 570578 (2017)

13. H. Shen, Improved threshold denoising method of mine video monitoring image in wavelet transform domain. Met Miner. 7(493), 151-154 (2017)

14. L. Xiaofei, Q. Xiaohui, An improved soft-threshold image denoising algorithm based on wavelet transform. Comp. Technol. Dev. 26(5), 7678 (2016) 
15. Z. Fengbo, L. Changgeng, H.-q. Zhu, Research on threshold improved denoising algorithm based on lifting wavelet transform in UV-vis spectrum. Spectrosc. Spectr. Anal. 2(38), 506-510 (2018)

16. Z. Xiaoyan, T. Abdukirimturki, Improvement of threshold image denoising algorithm with wavelet transform. Comp. Technol. Dev. 27(3), 81-84 (2017)

17. Anitha S, Subhashini $T$, Kamaraju M, A Novel Multimodal Medical Image Fusion Approach based on Phase Congruency and Directive Contrast in NSCT Domain. International Journal of Computer Applications. 129(10), 3035 (2015)

18. P. Sangeetha, S. Hemamalini, Dyadic wavelet transform-based acoustic signal analysis for torque prediction of a three-phase induction motor. IET Signal Proc. 11(5), 604-612 (2017)

19. A. Seal, D. Bhattacharjee, M. Nasipuri, et al., PET-CT image fusion using random forest and à-trous wavelet transform. Int. J. Numer. Methods Biomed. Eng. 34(3) (2017). https://doi.org/10.1002/cnm.2933

20. H. Tao, F. Xue, Q. Hongliang, et al., Remote sensing image denoising algorithm based on NSCT and adaptive fuzzy threshold. Comp. Technol. Dev. 1, 65-69 (2016)

21. X. Chu, Z. Zhou, G. Deng, et al., Study on damage identification of beam bridge based on characteristic curvature and improved wavelet threshold de-noising algorithm. Adv. Model. Anal. B 60(2), 505-524 (2017)

22. J. Cai, Y. Xiao, Impulse interference processing for MT data based on a new adaptive wavelet threshold de-noising method. Arab. J. Geosci. 10(18), 407 (2017)

23. Y. Xu, M. Luo, T. Li, et al., ECG signal de-noising and baseline wander correction based on CEEMDAN and wavelet threshold. Sensors 17(12), 2754 (2017)

24. P. Yang, L. Yang, X. Zhu, et al., Recognition method of speckle noise in interference fringe images based on object. J. Appl. Opt. (2017)

25. I.M. Sense, Supervised and unsupervised subband adaptive denoising frameworks with polynomial threshold function. Math. Probl. Eng. 2017(6), $1-12(2017)$

26. K. Zhou, S. Xing, C. Nie, A heart sound denoising method based on adaptive threshold wavelet transform. J. Guangxi Norm. Univ. (2016)

27. J.D. Zhu, J.L. Li, X.D. Gao, et al. Adaptive threshold detection and estimation of linear frequency-modulated continuous-wave signals based on periodic fractional Fourier transform. Circuits Syst. Sign. Proc. 35(7), 2502-2517 (2016)

28. H. Yao, L. Song, Construction method and application for threshold function family in wavelet threshold denoising. Int. J. Control. Autom. Syst. 9(8), 291302 (2016)

\section{Submit your manuscript to a SpringerOpen ${ }^{\circ}$ journal and benefit from:}

- Convenient online submission

- Rigorous peer review

- Open access: articles freely available online

- High visibility within the field

- Retaining the copyright to your article

Submit your next manuscript at $\boldsymbol{\nabla}$ springeropen.com 